\title{
A Successful Mechanical Thrombectomy for Acute Basilar Artery Occlusion in a Patient Who Had Undergone Fontan Operation
}

\author{
Yun Jik Park* (D), Hanim Kwon* (D), Kyu-Bong Lee* (D), Hana Lee ${ }^{\dagger}$, Jae-Suk Baek ${ }^{\dagger}$, Yunsun Song ${ }^{\ddagger}$ (D), \\ Sang-Beom Jeon* (D), Yeon-Jung Kim* (D), Eun-Jae Lee* (D) \\ Departments of Neurology ${ }^{\star}$, Pediatrics ${ }^{\dagger}$, and Radiology ${ }^{\ddagger}$, Asan Medical Center, University of Ulsan College of Medicine, Seoul, Korea
}

\begin{abstract}
Adults with a congenital heart disease (CHD) are increasing in number owing to improvement in diagnostics, medical management, and surgical techniques in infant and child patients. These patients are at increased risk of stroke; their improved longevity has increased the chance of adult neurologists encountering CHD patients. Adults with CHD may have distinctive stroke characteristics, because they have different hemodynamic features that last for a long time after corrective surgery. Herein we present the case of a female patient who had received Fontan operation due to functional single ventricle at the age of five. She developed sudden basilar occlusion at the age of 19 and was successfully treated with aspiration thrombectomy even after 24 hours of the symptom onset.
\end{abstract}

J Neurosonol Neuroimag 2019;11(2):154-157

Key Words: Acute stroke; Thrombectomy; Fontan procedure
Received: July 11, 2019

Revised: July 26, 2019

Accepted: August 3, 2019

Address for correspondence: Eun-Jae Lee

Department of Neurology, Asan Medical Center, University of Ulsan College of Medicine, 88 Olympic-ro 43-gil, Songpa-gu, Seoul 05505 , Korea

Tel: $+82-2-3010-1851$

Fax: +82-2-474-4691

E-mail: eunjae.lee@amc.seoul. $\mathrm{kr}$
Congenital heart disease (CHD) comprises a wide range of developmental cardiac problems, which include simple left and right shunt, obstruction, and mixing disorders resulting in cyanosis. Although its mortality rate was high ( $80 \%)$ in the 1950s, improvement in medical management and surgical techniques remarkably have expanded the life expectancy of these patients; nowadays more than $90 \%$ of children born with CHD reach adulthood. Fontan operation is a palliative surgical procedure performed in pediatric patients who have a functional single ventricle. ${ }^{1}$ Although the procedure is a life-saving operation, it inevitably induces long-term hemodynamic changes, which may confer a high risk of stroke. ${ }^{2}$ Moreover, these hemodynamic alteration may lead to a unique characteristics of stroke in patients treated with Fontan procedure. In this report, we present a case of 19-year-old female who had received Fontan operation due to functional single ventricle at the age of five and experienced acute basilar artery occlusion that was successfully treated with mechanical thrombectomy.

\section{CASE REPORT}

A 19-year-old woman with a history of CHD visited the emergency room, complaining of acute dizziness and ataxia. She underwent echocardiography for her cardiac murmurs at 1 month after birth and was diagnosed with functional single ventricle (complete atrioventricular septal defect with double outlet ventricle) and pulmonary stenosis. She received a total of seven cardiac surgeries, including Fontan operation at the age of five. After the surgery, she took warfarin in a daily basis. Although she revealed a low oxygen saturation $\left(\mathrm{SpO}_{2}\right.$ 80-95\%) at her usual time and regular outpatient visits (department of pediatric cardiology), she was doing well during daily living without significant discomfort.

At the night before the visit, she suddenly developed dizziness and gait ataxia so severe as to keep her from walking alone. When she woke up in the morning, the symptoms persisted and worsened; she found that her eyes did not move well. After lunch time, she also developed sensory deficits in the right hemi-body area, along with tingling sensation. 
Twenty hours after the symptom onset, she visited the emergency room. She showed 101/55 $\mathrm{mmHg}$ of blood pressure, $80 / \mathrm{min}$ of heart rate, and $37.0^{\circ} \mathrm{C}$ of body temperature, with 85 to $93 \%$ of oxygen saturation. Electrocardiography showed pacemaker rhythm and did not reveal arrhythmia such as atrial fibrillation. She was $143 \mathrm{~cm}$ tall and weighed $33 \mathrm{~kg}$. Mental status and orientation were alert and well preserved, respectively. On her eye examination, she revealed adduction limitation of the left eye, along with abducting nystagmus of the right eye, while on attempting rightward gaze. Remarkably, convergence was relatively spared. Based on these findings, we regarded that she developed internuclear ophthalmoplegia (INO). She did not have dysarthria or dysphagia. Her motor power in all the extremities were intact. Meanwhile, truncal ataxia was severely identified, as she was not even able to maintain the sitting position. On sensory examination, position and vibration sensations in the right side of the body were moderately decreased, as compared to those in the left side of the body. Pinprick sensations were also mildly impaired. Taken together, her initial National Institute of Health Stroke Scale (NIHSS) was 3.

Laboratory tests showed mild anemia (11.1 g/dL), normal platelet counts $\left(326 \times 10^{3} / \mathrm{mL}\right)$ and normal blood glucose level (106 mg/dL). Of note, prothrombin time (international normalized ratio [INR], 1.60) did not stand in the therapeutic range (INR 2-3).
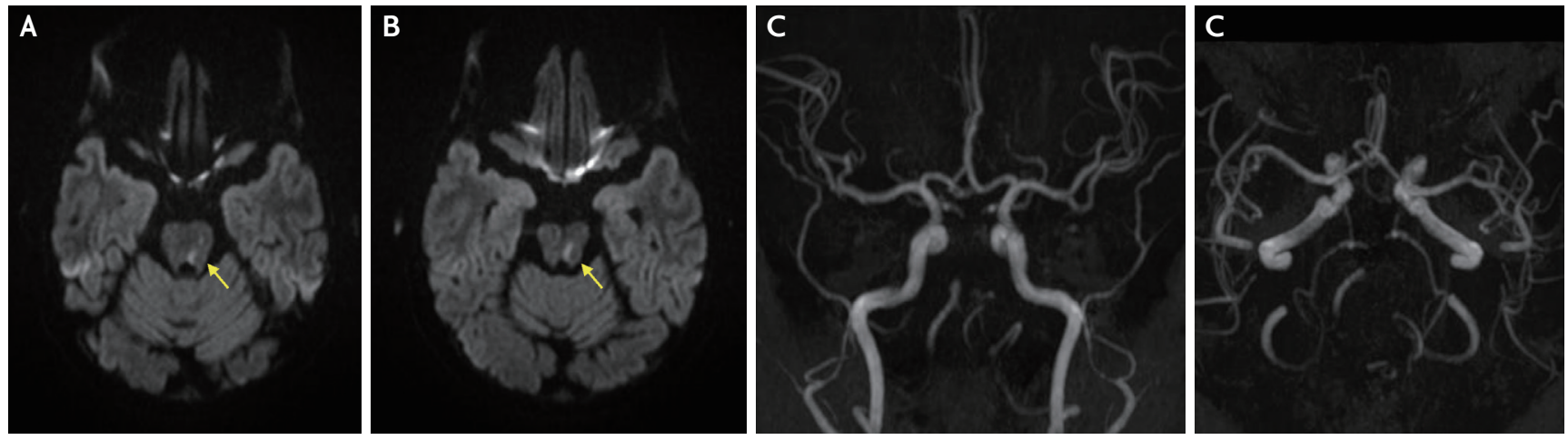

FIG. 1. Brain magnetic resonance imaging (MRI) findings. (A, B) Initial brain MRI revealed acute infarction in left dorsal pons on diffusion-weighted imaging (yellow arrows). (C) Time-of-flight magnetic resonance angiography basilar artery occlusion. (D) The flow in bilateral posterior cerebral arteries was preserved via collateral flow from posterior communicating arteries.
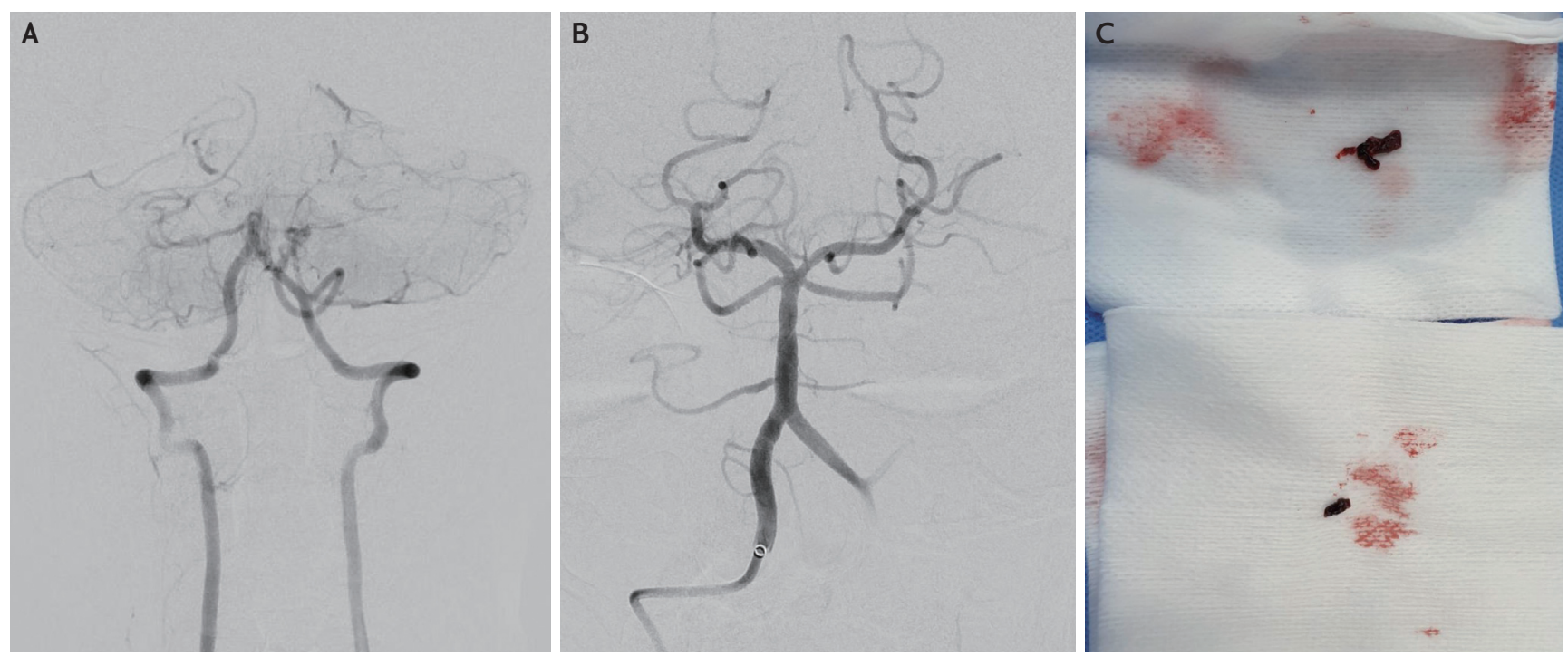

FIG. 2. (A) Transfemoral cerebral angiography findings and mechanical thrombectomy. (B) Cerebral angiography revealed total occlusion of proximal basilar artery. (C) After aspiration thrombectomy, basilar artery was recanalized and large red clots were retracted. 
Diffusion-weighted imaging, performed at 25 hours after the symptom onset, showed a focal high signal intensity in the left dorsal pons (Fig. 1A, B). However, acute lesions correspondent to her severe whirling-type dizziness and truncal ataxia were not observed, suggesting a clinical-diffusion mismatch. Accordingly, magnetic resonance angiography revealed basilar occlusion with bilaterally prominent posterior communicating arteries (Fig. 1C, D).

Although neurologic deficits, defined by NIHSS, were not much severe, we decided to try mechanical thrombectomy considering a progressively worsening clinical course and a definite clinical-diffusion mismatch. Cerebral angiography, performed at 28 hours after the symptom onset, demonstrated basilar artery occlusion (Fig. 2A). Clot removal was tried couple of times, and red clots were taken out from the basilar artery, recanalizing the occluded segment (Fig. 2B, C).

After the aspiration thrombectomy, her neurologic deficits improved rapidly. At 4 days after the procedures, she could walk alone without significant gait ataxia; at 20 days, her abnormal eye findings (internuclear ophthalmoplegia) were also normalized.

\section{DISCUSSION}

We described a case of successful mechanical thrombectomy for acute basilar occlusion in patients with a history of Fontan operation. Although the risk of stroke in patients with Fontan operation has been suggested, ${ }^{2,3}$ treatment with a mechanical thrombectomy in these patients has been rarely reported. ${ }^{4}$ Clot-retrieval strategies in acute stroke settings, the proven treatment option by the guideline, may also be feasible in stroke patients with CHD who underwent the corrective surgery.

Fontan operation is a surgery performed in children with univentricular hearts (e.g., univentricular congenital heart lesions, tricuspid atresia, and double inlet left ventricle). The single ventricle exerts nearly twice the expected amount of work, because it should pump out blood for both the body and lungs; hence it is prone to be dysfunctional resulting in cyanosis, pulmonary hypertension, and heart failure. ${ }^{5}$ Fontan operation creates a circulation that diverts the venous blood from the inferior vena cava and superior vena cava to the pulmonary arteries via the right atrium, without passing through the morphologic ventricle.

Although decreasing the ventricular burden, this procedure induces complex, long-term hemodynamic changes. ${ }^{1,2}$ The abnormal ventricular morphology per se contributes to ventricular dysfunction. In addition, prolonged exposure to elevated intracardiac pressures gives rise to atrial dilatation, fibrosis, and subsequent development of cardiac dysrhythmia. Meanwhile, the low and non-pulsatile cardiac output after Fontan operation may result in impaired nitric oxide release and subsequent endothelial pathway dysfunction, elevating systemic and pulmonary vascular resistance. Finally, portal hypertension induced by Fontan operation may cause ascites, cirrhosis, and protein-losing enteropathy, resulting in coagulopathy. All of these factors may contribute to the occurrence of stroke. ${ }^{1,2}$ The guideline recommends the consideration of long-term anticoagulation in adults with Fontan palliation even without known or suspected thrombus, thromboembolic events, or prior arrhythmia (class IIb). ${ }^{6}$ Although our patient had taken warfarin regularly, the INR level was not within therapeutic range at symptom development.

Remarkably, this patient underwent mechanical thrombectomy more than 24 hours after the symptom onset. Currently, the mechanical thrombectomy with stent retrievers are recommended up to 6 hours after stroke onset and even up to 24 hours after stroke onset using advanced brain imaging. ${ }^{7}$ However, the only evidence of the intervention for effectiveness and safety in randomized trials exists for large artery occlusion in anterior circulation stroke. Therefore, although mechanical thrombectomy appears to be safe and effective in patients with basilar artery occlusion, ${ }^{8}$ its application to posterior circulation stroke in should be individualized considering relevant clinical factors. In this case, a deteriorating clinical course and a clinical-diffusion mismatch were the factors considered in determining the decision to conduct interventions. Moreover, her young age and a grave prognosis that may happen if strokes occur fully across the basilar arterial territory were also regarded.

Then why were not symptoms so much severe with basilar occlusion even 24 hours after the symptom onset? First, the salvageable brain tissues per se may be 
more abundant in the posterior circulation than in the anterior circulation; brain tissues in the former territory contain a higher proportion of white matter (in the brainstem) and better collateral arterial flow. ${ }^{9}$ Second, this patient may have experienced long-term preconditioning training against cerebral ischemia. ${ }^{10}$ Her usual oxygenation level was suboptimal with a range between 80-95\%. Although hypothetical, this stressful condition may have trained and enhanced her tolerance against ischemic attacks.

In conclusion, clinicians should be aware of that patients with a history of Fontan operation may be at risk of stroke due to altered hemodynamic process, and that acute mechanical thrombectomy may be an effective and feasible treatment for CHD patients who underwent corrective surgery.

\section{Acknowledgments}

This work was supported by Ministry of Science and ICT (NRF-2018M3A9E8066249), Korea.

\section{Conflicts of Interest}

No potential conflicts of interest relevant to this article was reported.

\section{REFERENCES}

1. McRae ME. Long-term issues after the Fontan procedure. AACN Adv Crit Care. 2013;24:264-282.

2. Smith JMC, Andrade JG, Human D, Field TS. Adults with complex congenital heart disease: cerebrovascular considerations for the neurologist. Front Neurol. 2019;10:329.
3. Driscoll DJ, Offord KP, Feldt RH, Schaff HV, Puga FJ, Danielson GK. Five- to fifteen-year follow-up after Fontan operation. Circulation. 1992;85:469-496.

4. Savastano L, Gemmete JJ, Pandey AS, Roark C, Chaudhary N. Acute ischemic stroke in a child due to basilar artery occlusion treated successfully with a stent retriever. J Neurointerv Surg. 2016;8:e33.

5. O'Leary PW. Prevalence, clinical presentation and natural history of patients with single ventricle. Prog Pediatr Cardiol. 2002;16:31-38.

6. Stout KK, Daniels CJ, Aboulhosn JA, Bozkurt B, Broberg CS, et al. 2018 AHA/ACC guideline for the management of adults with congenital heart disease: a report of the American College of Cardiology/American Heart Association task force on clinical practice guidelines. Circulation. 2019;139:e698-e80o.

7. Nogueira RG, Jadhav AP, Haussen DC, Bonafe A, Budzik RF, Bhuva P, et al. Thrombectomy 6 to 24 hours after stroke with a mismatch between deficit and infarct. $N$ Engl J Med. 2018;378:11-21.

8. Weber R, Minnerup J, Nordmeyer H, Eyding J, Krogias C, Hadisurya J, et al. Thrombectomy in posterior circulation stroke: differences in procedures and outcome compared to anterior circulation stroke in the prospective multicentre REVASK registry. Eur J Neurol. 2019;26:299-305.

9. Lindsberg PJ, Pekkola J, Strbian D, Sairanen T, Mattle HP, Schroth G. Time window for recanalization in basilar artery occlusion: speculative synthesis. Neurology. 2015;85:18061815 .

10. Dirnagl U, Becker K, Meisel A. Preconditioning and tolerance against cerebral ischaemia: from experimental strategies to clinical use. Lancet Neurol. 2009;8:398-412. 